\title{
Methods for Dichoptic Stimulus Presentation in Functional Magnetic Resonance Imaging - A Review
}

\author{
Bhaskar Choubey*,1,2,3, Alina Jurcoane $e^{2,3,4,5}$, Lars Muckli ${ }^{3,4,6}$ and Ruxandra Sireteanu ${ }^{2,3,5,7}$ \\ ${ }^{1}$ Department of Engineering Sciences, University of Oxford, Oxford, OX1 3PJ, UK \\ ${ }^{2}$ Department of Biological Psychology, Institute for Psychology, Johann Wolfgang Goethe-University, Mertonstrasse 17, \\ 60054 Frankfurt, Germany \\ ${ }^{3}$ Department of Neurophysiology, Max-Planck-Institute for Brain Research, Deutschordenstrasse 46, 60528 Frankfurt, \\ Germany \\ ${ }^{4}$ Brain Imaging Centre, Schleusenweg 2-6, Haus 95H, 60528 Frankfurt, Germany \\ ${ }^{5}$ Centre for Research on Individual Development and Adaptive Education (IDeA), Frankfurt, Germany \\ ${ }^{6}$ Centre for Cognitive Neuroimaging, Department of Psychology, University of Glasgow, 58 Hillhead Street, Glasgow \\ G12 $8 Q B, U K$ \\ ${ }^{7}$ Department of Biomedical Engineering, College of Engineering, Boston University, 44 Cummington Street, Boston, \\ Massachusetts 02215, USA
}

\begin{abstract}
Dichoptic stimuli (different stimuli displayed to each eye) are increasingly being used in functional brain imaging experiments using visual stimulation. These studies include investigation into binocular rivalry, interocular information transfer, three-dimensional depth perception as well as impairments of the visual system like amblyopia and stereodeficiency. In this paper, we review various approaches of displaying dichoptic stimulus used in functional magnetic resonance imaging experiments. These include traditional approaches of using filters (red-green, red-blue, polarizing) with optical assemblies as well as newer approaches of using bi-screen goggles.
\end{abstract}

Keywords: Dichoptic visual stimulation, fMRI, filters, video goggles.

\section{INTRODUCTION}

Functional magnetic resonance imaging (fMRI) has evolved as an important method to study brain function providing several new insights into our understanding of the neural physiology. fMRI experiments measure the brain activity in a subject performing a pre-decided task, generally in response to external stimuli. Visual stimuli have formed an important set of external stimuli for many of these experiments and have been used to study almost every brain function.

The majority of experiments require the display of the identical stimuli to both eyes. However, an important group of experiments has evolved over the last few years involving different stimuli displayed to the two eyes leading to dichoptic presentation of stimuli. For examples, such stimulus has been extensively used in studies into binocular rivalry [1-3], interocular information transfer $[4,5]$, three-dimensional depth perception [6-8] as well as investigations into impairments of the visual system like amblyopia [9-11], and stereodeficiency [12].

\footnotetext{
*Address correspondence to this author at the Department of Engineering Sciences, University of Oxford, Oxford, OX1 3PJ, UK; Tel: +44 18652 73805; Fax: +44 18652 73905; E-mail: bhaskar@ robots.ox.ac.uk
}

The need to display dichoptic visual stimuli has led to development of various tools and stimuli over the years. In this paper, we review these methods leading to a description of a technique developed by us to display the stimuli for experiments in interocular studies. Section II describes the dichoptic stimuli in detail and provides an overview of the different approaches applied in the presentation of these stimuli. The simplest and most common approach of fused images is analyzed in Section III. Non-fused image approaches including special purpose assemblies designed for dichoptic stimuli are discussed in Section IV. We also introduce our methods of bi-screen goggles in this section. Section $\mathrm{V}$ explains various considerations while using bi-screen goggles along with an example of their use in our experiments. Section VI provides the conclusion. This paper is devoted primarily to dichoptic stimuli; however, the discussions related to display systems are generally valid to all optical stimuli in fMRI experiments.

\section{DICHOPTIC STIMULI}

Blending the images of the two eyes into one single percept is one of the most fascinating feats of the human brain. Over the years, researchers have attempted to understand the nature of the different aspects of binocular interaction, like binocular single vision, fusion, stereoscopic depth perception, binocular contour rivalry, eye dominance and the clinical aspects of interocular suppression and amblyopia. Sev- 
eral devices were developed in past centuries, culminating with Wheatstone's mirror-based stereoscope (for a comprehensive historical overview, see Wade [13]). The interest persists to the present day, and is manifest in different approaches to investigate the brain mechanisms underlying the combination of the information coming from the two eyes. These studies range from investigations on the mechanisms of stereoscopic vision $[14,15]$ through the investigation on the cortical site of binocular rivalry suppression, which serves as a model for (un)conscious perception $[2,16,17]$ to the mechanisms leading to the progressive elimination of one eye from conscious perception in anisometropic [18] and strabismic amblyopia [11]. The prerequisites for approaching these questions are a flawless isolation of the two monocular pathways, paired with a smooth transition between the two monocular percepts.

With the advent of functional neuro-imaging, new vistas were opened in our approaches to understanding the visual system. However, display mechanism in these experiments is severely limited on account of supine position of the subject, limited volume around the head in the imaging tube as well as restrictions on MRI compatible instrumentation on account of the high magnetic field. This demanded modifications of the traditional techniques or development of new techniques for dichoptic display in these experiments. A lack of standardisation in experiments protocols, however, has led to several techniques and instrumentation thereof.

Nevertheless, it is possible to broadly classify these techniques in two groups based on the display instrument. These are projector-mirror-based and goggle-based. In the more popular, external projector technique, the images are projected onto a passive screen placed near the end of the subject bed in the MRI machine [2, 11, 19, 20]. The images are stored in a computer and displayed onto a screen inside the imaging room by the projector. The computer and the projector are placed in a room outside the imaging room. This ensures that the computer and the projector do not have to be MRI compatible, thereby reducing cost. The most popular display in this technique is one placed at the end of the subject bed forming a back-projection system. The subject is able to view the stimulus on the screen using an assembly of mirrors placed in his/her head coil [20]. In a slightly modified technique, a computer display covered by a Faraday cage may be placed at the end of the subject bed and the subject may again view the stimuli using the mirror assembly $[4$, 21]. It is, however, possible to avoid the mirror using a fiberscope [22].

In the second group of techniques, the display device is placed next to the subject's eye below the headcoil. This display device generally takes the form of bi-screen goggles [23-25]. Dichoptic stimuli can be presented to the subject using either of these methods.

Yet another way to classify the display the dichoptic stimulus could be based on the nature of image being projected or displayed. In one category, the two stimuli are fused to form one image presented to the subject. The subject differentiates between the two using spectral or polarization filters. In the second approach, the two images are presented independently to the subject without fusing. Two different optical paths are ensured to remove optical crosstalk between the stimuli. In the next few sections, examples of both of these techniques will be presented and analyzed to identify the optimum approach to dichoptic stimuli.

\section{FUSED IMAGE APPROACHES}

In the simplest form of stimulation, only one eye needs to be stimulated during one run of the experiment. In these experiments, one performs the whole experiment by patching one of the eyes and a repeat experiment by patching the other eye [26]. Another approach is to use differently coloured stimuli for each eye $[10,11]$, while the subject wears coloured glasses of different spectral pass-bands for each eye. Thus, the subject is capable of viewing images of any one colour only. The images are still projected through the external projector onto the screen. However, a set of colour filters is also placed in front of the projector's lens as shown in Fig. (1C). The monochrome image produced by the projectorfilter assembly has the spectral components of only those colours which are passed through the filter in front of the projector. This ensures that images corresponding to only one colour are displayed on the screen and hence only one eye of the subject is stimulated at any time. For binocular stimulation, the spectral filter in front of the projector is replaced by a neutral density filter.

The aforementioned approaches are simple and cheap to implement; however, they fail to produce true dichoptic stimulation as only one of the eyes is stimulated at any instance. One way to produce a pseudo-dichoptic stimulus
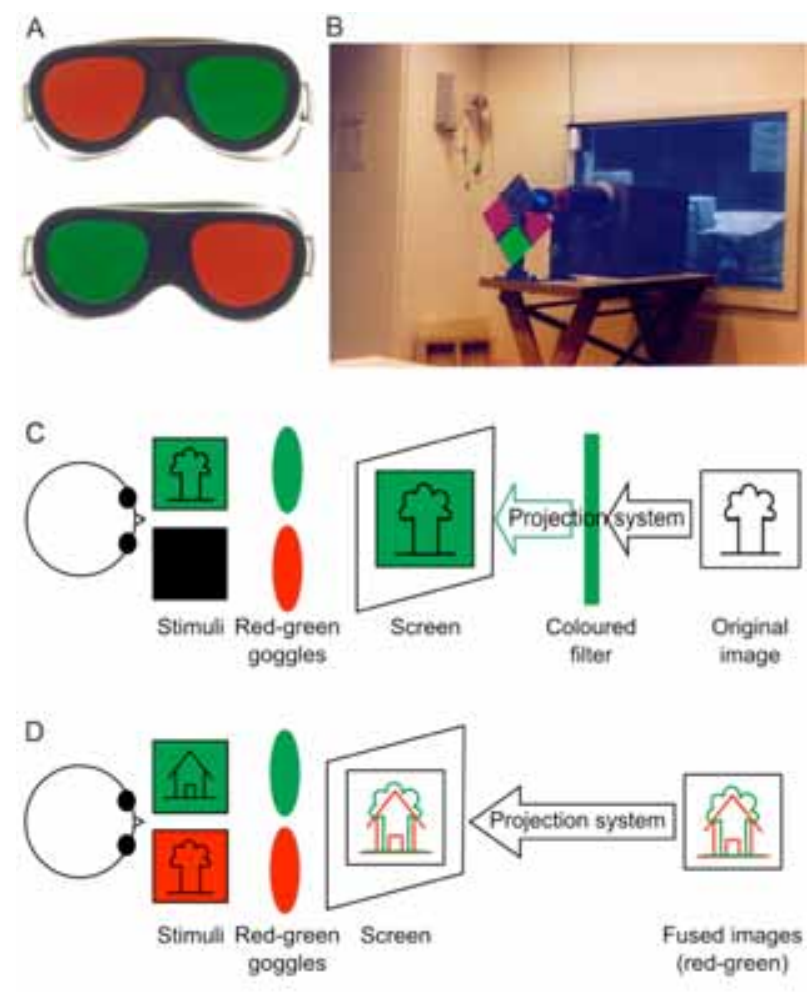

Fig. (1). Coloured filters are the most frequently used method for dichoptic visual stimulation. During the experiment, the subject views the stimuli through a pair of coloured red-green goggles (A). The light reaches the viewing screen either through a coloured filter standing in front of the projector (B and $\mathbf{C}$ ) or red/green stimuli are directly projected onto the screen (D) (Method first described by Sireteanu and co-workers (1998) [9]. Photographs made by Natalie Tonhausen). 
could be to rotate the colour filter in front of the projector at a high speed. However, this would require a fast mechanical system and hence is not the preferred choice for these stimuli. Researchers have hence utilized the technique of image fusion to remove the filter assembly in the projection path. In this technique, the two stimuli are generated with different filterable properties, either in spectral or in polarization domain. These are then fused to form a single image.

The stimulus flow diagram of Fig. (1D) shows one such setup using stimuli of red and green colours. In this setup, the fused single image is displayed on the screen inside the scanner room using the external projector. The subject wears filters of the appropriate kind over his eyes to differentiate these images. Monocular or binocular stimulation may be achieved by simply manipulating the colours presented on the screen. Image fusion techniques have been used extensively in classical psychophysical experiments and are hence properly characterized. This also means that several psychophysical experiments could be converted into neuroimaging experiments with considerable ease. The comparison between the two also becomes a straightforward process. These advantages have hence, led to rapid and extensive use of these techniques by the imaging community.

\section{A. Coloured Filters}

Among the spectral separation techniques, red-green filters (Fig. 1) have been the filters of choice [1, 9, 11, 27-29]. Nevertheless, red-blue filters have also been used by some researchers [30-33]. These filters have found use in studies involving the whole spectrum of dichoptic stimulus including study of binocular rivalry, depth perception, invisible stimulus perception and studies of amblyopia.

Lumer and co-workers have used a red-coloured drifting grating shown to one eye and a green-coloured face shown to the other eye to study perceptual rivalry [1]. Several other groups have investigated binocular rivalry with the help of these red-green filters at different levels on the visual processing pathway. For example, Tong and co-workers have used this techniques to study human extrastriate cortex [2] as well as in the cortical representation of the blind spot [34], Lee and co-workers in V1 [35], Wunderlich and co-workers in LGN [29]. Similarly, amygdala's response to different facial expressions under binocular suppression has been studied using superimposed images of red and green faces and houses by Williams and co-workers [20]. Moutoussis and co-workers have used red-green houses and faces to study the role of invisible stimuli in cortical activation [36]. They have also used drifting random dot patterns for studying binocular rivalry in motion perception [16]. The role of visual phantoms using binocular rivalry has also been studied using red-green stimuli by Meng and co-workers [37].

Yet another use of red-green has been to investigate visual disparity by Tsao and co-workers [14] while red-green stereo images have been used to study shape selective visual processing by Gilaie-Dotan and co-workers [19]. Stereoscopic depth perception in visual cortex has been investigated by Brouwer and co-workers using slanted wire-frame drawings and stereo-photographs of natural scenes, objects, and faces using red-green filters [27].

Investigations into impairments of the visual system, including amblyopia, have also been conducted using red- green filters. Sireteanu and co-workers have used red-green stimuli to study the primary visual cortex in amblyopic subjects $[9,11]$ while Liu and co-workers have investigated eye dominance in the visual cortex and LGN activation in patients with amblyopia [10, 18].

Amongst the users of red-blue filters, Haynes and coworkers investigated the binocular rivalry in LGN [17]. They have also attempted to predict the stream of consciousness in human visual cortex using red-blue horizontal and vertical grating stimuli [32]. Negawa and co-workers have used coloured random-dot stereograms with red-blue filters to study the role of M-pathway and area 44 and 45 for stereoscopic perception [30]. Pasley and co-workers have utilized redblue videos of moving houses to study the discrimination of unperceived objects during binocular rivalry [31]. In yet another experiment, Naganume and co-workers have used red-blue images to present binocular disparity cues to the brain to investigate processing of geometrical shapes [33].

\section{B. Polarized Filters}

Another approach to separate fused images has been to use different polarisation in individual images rather than colour [4, 38-40]. For example, Rutschmann and co-workers have used a mirror-prism assembly along with an LCD projector and polarizing filters to generate virtual depth planes $[39,40]$. They claimed that their particular setup ensured no crosstalk between stimuli for the left and right eye. These stimuli were used to investigate the dorsal visual pathway in motion processing and its role in motion processing. In another experiment, Büchert and co-workers have used the same assembly and dichoptic checkerboard stimuli to further explore binocular interactions [4, 41]. Another related study, although not an fMRI study, is that by Gulyas and Roland, who have used polaroid spectacles in a functional PET study of visual cortex' ability to discriminate the binocular disparity [38]. Another use of polarised filters has been by Taira and co-workers who have used a 21-in display with a liquid crystal polarized filter [42]. This display was placed in front of the subject at eye level. To display the stimuli, they switched the filter at double frame frequency, at $120 \mathrm{~Hz}$. The filter was switched with each frame of the display so as to display 60 frames/s of stimulus to each eye. The subjects wore polarized glasses to view these stimuli stereoscopically.

\section{Discussion}

Use of fused images, despite being a simple and timeproven technique, is prone to errors and limitations. A sever limitation arises when the pixels from the two independent stimuli fall on exactly the same position on the screen, as observed by Moutoussis and co-workers. The solution as proposed by them is to makes these pixels yellow in order to be visible to both eyes $[16,36]$.

The principal demerit of the spectrally separated images, however, lies in the monochrome nature of the final stimulus. This severely limits the range of visual experiments. Polarized filters do not suffer from this limitation. However, they are prone to at least two other inconveniences. First, their function as neutral density filters attenuates the light signal leading to low illuminations in the imaging room, which is otherwise fairly dark and hence compounds the problem. Secondly, their effect of dichoptically separating 
two images is highly dependent on the angulation of the head of the subject (implicitly the angulations of the headmounted polarized goggles). Even small head movements may cause large cross-talk between the stimuli (Fig. 2).

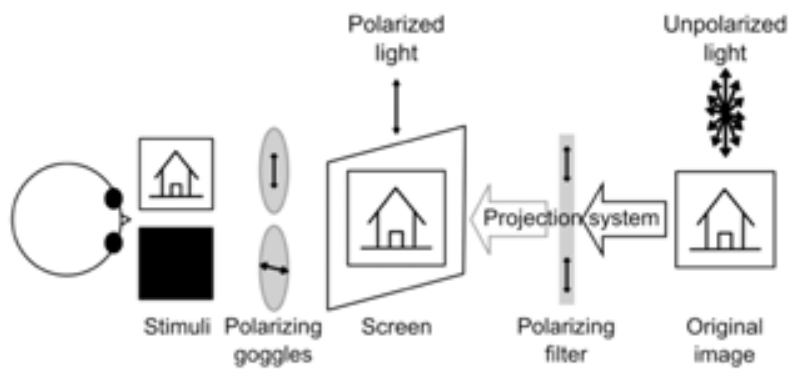

Fig. (2). Simplified sketch of one possible experimental setup involving polarized goggles and filter. During the experiment, the subject wears a pair of polarizing goggles while the light coming from the projector is polarized through a filter. If the two polarizing devices (filter and goggles) polarize light in orthogonal planes, no light reaches the eye (i.e. right eye). This does not happen if the planes are parallel (i.e. left eye) and all light reaching the goggles passes through. For intermediate angles between the polarizing planes, various amounts of light reach the eye (not shown).

An additional problem with filter-based binocular stimuli is that that one has to repeat the experiments with the position of the filters reversed so as to remove the filter induced effects, if any. For example, with red-green colour filters, one has to perform one set of experiments with a red filter on the left eye and a green one on the right eye. Another set of experiments has to be performed with red filter on right eye and green on left eye. There are three possible ways to perform the repeat experiment with reversed position. In the first, both sets of experiments may be performed with the same subject during the same scan. This would severely limit the length of the experiment owing to the limited time a subject can be placed inside a scanner. To counter this, one may use two different scanning sessions for the same subject. A third strategy applied is to use a large number of subjects with half of them wearing the red filter on the left eye and other half wearing the red filter on the right eye. All these techniques, however, increase the experiment time.

Despite these limitations, imaging researchers have successfully adapted the traditional psychophysical techniques to fMRI environments, using projectors and mirror assemblies. However, these adaptations come at a cost of involved complexities. The optical setting of prism and mirrors is not always flawless. Any optical misalignment leads to indeterminable errors in the brain activation. These methods are also very laborious and require a great deal of experimental preparation, making them rather inconvenient for routine scanning.

Even more concerning is the use of projectors. The LCD technique used in most projectors is prone to several drawbacks affecting the dichoptic stimulus. The first of these are the drastic variations in luminance and contrast characteristics across the screen as well as across the grey levels [43, 44]. These non-uniformities lead to errors in brain activation as reported by Strasburger and co-workers [45, 46]. They observed lateralized activation in the primary visual cortex when stimulating with Gabor patches on a standard LCD setup. On further investigation, these were found to be caused by non-uniformity of the stimulus being presented. They have also shown that errors are further exaggerated due to non-uniform and non-linear gamma function of the screen. This leads to bright parts of the image being overproportionally brighter and the dark parts not dark enough. Strasburger and co-workers have suggested luminance homogenization as well as position dependent gamma correction to correct for the artefacts introduced by these defects. However, these steps are time consuming, with the reported correction time being 6 hours.

Yet another drawback of these systems has been identified by Wiens and co-workers [47, 48]. They show that LCD/TFT shows poor characteristics when displaying the stimulus at brief durations and hence form a poor source in visual masking experiments. One of the earliest works in the field of stimulus display, by Cornelissen and co-workers has noted few other drawbacks while using projectors. These include a restricted field of view (due to the long aspect ratio of the bore), difficulty in characterizing the image quality (which is highly dependent on viewing angle) and susceptibility to loss of contrast due to stray light reaching the projection screen [22].

\section{NON-FUSED IMAGE APPROACHES}

These limitations have led to development of approaches without the need of fusing the two images in dichoptic stimuli. Two different routes have been attempted. In the first, attempts have been made to project two different images and hence remove the need of fused images. However, a projector-based assembly is still used. In the second approach, the projectors have been replaced completely by other display devices.

\section{A. Projector-Based}

In order to avoid the use of fused images in projector assemblies, some researchers have developed special purpose instrumentation for dichoptic presentation of stimuli. One such setup includes custom stereoscopes made of various mirror/prisms used by Büchert and co-workers to study the binocular interaction [4].

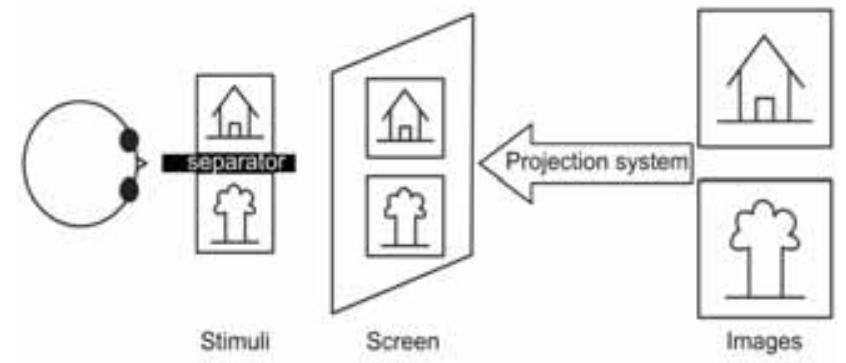

Fig. (3). Schematic drawing of a projector based custom stereoscope.

In these stereoscopes, two images are projected using an assembly of mirrors inside the scanner to differentiate the images (see Fig. 3). The stimulus is still presented to the subject through back-projection with an LCD projector; however, the screen is divided into two half screens. An assembly of prisms and polarized filters is used to provide two different images on these two half-screens thereby ensuring 
dichoptic stimulation. A similar approach has also been used by Merboldt and co-workers, while investigating stereoscopic depth perception [8]. They use a modified projection assembly by placing a semi-transparent screen fixed directly atop the headcoil of the MRI system. The projector placed outside the room is used to project onto this display using a lens placed at the end of the patient table. Mirrors and oculars were used for each eye to differentiate the images.

Another example of a purpose-built system is that of Nishida and co-workers [7, 49, 50]. Their assembly uses two sets of projection and display equipment including image guides, LCD projectors, C-mounted objective lenses and eyepieces. The image signals are generated by two personal computers and projected by the respective LCD projectors. These signals are then sent to the eye-piece through the image guides, both of them being diamagnetic. The subjects in the MRI magnet could see the images through the eyepieces located in front of their eyes. The two images are hence separated from origin leading to a dichoptic visual stimulus. They have primarily used this system to study stereopsis.

A similar product is also available commercially from Avotec [51, 52]. It consists of two LCD projectors with different fibre-optic channel-leads to reach the different eyes. The claimed resolution for each LCD is 800 (horizontal) $\mathrm{x}$ 225 (vertical) with a field of view (FOV) of 30 (horizontal) $x$ 23 (vertical) degrees of visual angle focused at 2 dioptres. Analysing similar systems, Logothetis and co-workers have found the effective resolution being determined by the fibreoptic projection system, the fibre optic bundle being made of $530 \mathrm{H} \times 400 \mathrm{~V}$ fibbers [52].

\section{B. Non-Projector Based}

One of the earliest critics of LCD projector-based assemblies originated from the works of Cornelissen and coworkers [22]. In order to avoid many of the disadvantages of LCD displays/projectors described at the end of the previous section, they devised a binocular fiberscope consisting of three parts: an objective lens that images the world (in their case a CRT display), a fibre optic image guide and an eyepiece through which the image is viewed. It may be observed that aforementioned approaches of dividing the stimulus from its origins, as by Nishida and co-workers $[7,49,50]$ as well as Avotec assembly use similar fibre-optic channels. However, fibre optic approaches introduce additional errors on account of long image guides that attenuate the luminance of the image, especially at shorter wavelengths (giving the white CRT image a yellowish cast). Further, contrast loss due to light leakage between fibbers has also been reported. Additionally, glass optical fibbers are very sensitive to mechanical manipulation, breaking if bent too sharply, thereby resulting in black spots in the image (pixel dropout).

In another approach similar to those of Büchert et al. [4] and Merboldt et al. [8], Neri and co-workers have placed a flat-panel LCD display covered in a Faraday cage just beyond the end of the scanner bed [21]. They then use an assembly of two angled mirrors and binoculars to separate the images. Two monocular images are then displayed on the left and right halves of the LCD display respectively. Using a septum placed near the subject's knees and the adjustment of the mirrors, they were able to ensure that the stimulus is dichoptically presented. This assembly has been used to in- vestigate binocular rivalry as well as depth perception by several other groups including those of Polonsky [3], Backus [6] and Lee [35, 53].

Our interest in dichoptic stimulation originates from our research into interocular transfer phenomena and amblyopia $[5,9,11,12,23]$. In the past, we have used red-green glasses for display of these stimuli $[9,11]$. Owing to several limitations of the fused images approach described earlier, we investigated better options to present various stimuli. In addition, we also sought to devise an easy and a general-purpose setup with minimal optical configuration required. This means that any out-of-imaging-room projector-based or endof-table display-based assembly did not meet our ideal requirements. The simplest system would be one which can position a small-size display in front of the subject's eyes. Further, two different displays with preferably two different pathways for the right and left eye stimuli would be required (Fig. 4).

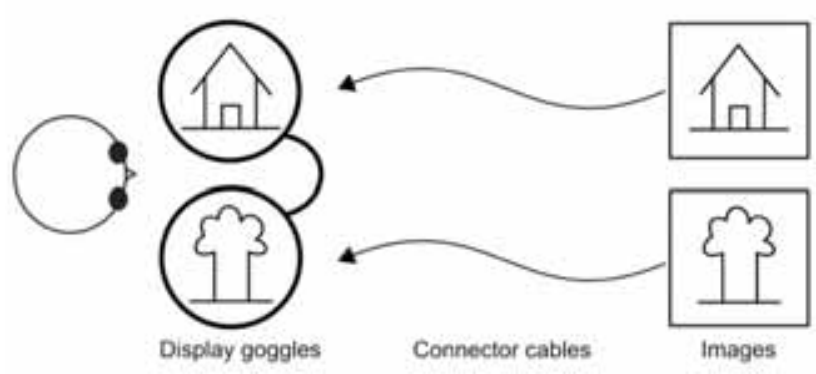

Fig. (4). Stimulus diagram using bi-screen goggles.

One such device, which could meet both these requirements, was the bi-screen goggles, and hence we investigated them for our experiments. Studies using goggles for stimulus display in fMRI have already been reported in past [54-56]. Firms including Resonant Technologies [25] and Nordic NeuroLab [24] are offering MRI compatible video goggles. These goggles behave as two LCD screens placed in front of the eyes and hence do not require the complicated optical assemblies needed in projector systems. Optional eye-tracker modules can also be attached to such devices. We selected VisuaStimDigital goggles (Fig. 6) from Resonant Technologies [25] owing to their small size which made it possible to place them inside an 8-channel head coil. We have used these goggles with success in experiments relating to interocular transfer of adaptation [5] as well as vision deficiencies $[12,23]$. In the next section, the experimental setup for the dichoptic stimulation using the goggles as well as the hardware and software requirements is described. The rest of this section, however, will be devoted to a comparison of the various techniques discussed.

\section{Discussion}

Classical assemblies using projectors and filters have widespread use. The goggles are fairly new as far as use of display medium in fMRI experiments is concerned. However, requirements of an optical setup make the classical approaches more difficult to use than goggles. Goggles do require special-purpose hardware support in the form of the goggles themselves and proper driving hardware. In addition, they also require some special-purpose software support. On the other hand, the software required for using fused 
images has been developed over time and is fairly mature. In addition, the goggles are currently more costly than simple optical assemblies. Nevertheless, goggles are simpler to use compared to any other system and provide feasibility for a broader spectrum of experiments than coloured glasses or polarized filters. Further, they are preferable to other nonfused approaches owing to their much simpler setup.

A recent comparative study between goggles and projectors as display medium is worth mentioning here. Engström and co-workers have performed a visual stimulus experiment twice, once with projector-based assemblies to display the stimuli and on a subsequent run, using a video goggle placed inside the head coil to display the stimuli [57]. They report that the stimulus shown through the projector fails to excite some parts of the brain. Their study adds further support to the use of goggles, in addition to simplicity.

\section{TWO SCREEN GOGGLES}

\section{A. Example Experiment}

One experiment where we used these bi-screen goggles has been in investigating the interocular transfer of information in several areas of the human brain $[5,12,23]$. To test the existence of interocular transfer of adaptation, we needed to stimulate the two eyes independently; therefore a need to present the stimuli in a dichoptic manner. These stimuli, shown in Fig. (5), are monochromatic and are presented at moderately high temporal frequencies.

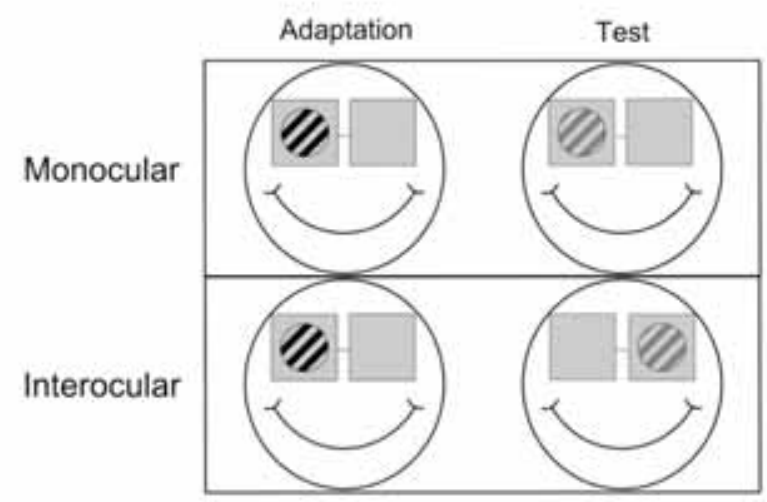

Fig. (5). Sketch of an interocular transfer of adaptation paradigm. In the first step one eye is adapted (left) and in the second step adaptation is tested (right) either in the same (top row) or the other eye (bottom row) (figure source Jurcoane et al. 2007 [12] - modified).

However, our aim in devising the display system was to make it as general purpose as possible, which can be used in future experiments involving colour, depth perception and apparent motion. We further strived to minimize any optical assemblies and complicated setups. The optical goggles we used are shown in Fig. (6).

These goggles can be used in two modes. One of the modes is used to display the same stimulus to both screens (mono) while the other can be used to separately program the two screens (stereo) leading to a dichoptic stimulus. Both displays each have a horizontal field of view of 30 degrees and a maximum resolution of $800 \times 600(\mathrm{SVGA})$. Further, lenses are also provided to correct refraction errors in various

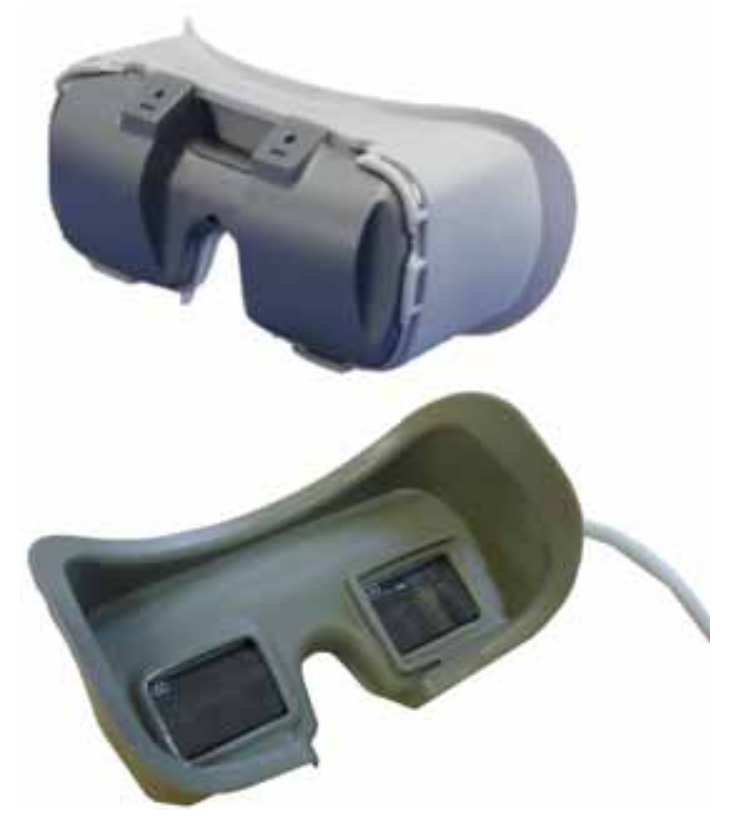

Fig. (6). Bi-screen goggles (VisuaStim Digital - Resonance Technologies, Northrige, USA).

subjects. It must be noted, however, that additional considerations may be needed when using these goggles in various experiments.

\section{B. Hardware Requirement}

In addition to the MRI-compatible two-screen goggles, the display system also requires a graphic card capable of driving the two screens (dual head graphic card). An alternate solution may be to use two separate graphic cards for the display. Dual head graphic cards are standard components in the market with various options of memory and resolution available. We have used a dual head Sapphire Radeon 9200SE graphic card with $128 \mathrm{Mb}$ memory. With different experiments, one should account for the right amount of graphic card memory required to enable display of all stimuli without any inter-frame disruptions.

The graphics cards available in the market generally offer several options of resolutions on the output displays. However, use of these options is limited by the maximum resolution provided by the goggles systems. Most goggles systems designed for MRI-usage have the SVGA resolution of $800 \times 600$ to match the resolution of LCD projectors. However, the optimum resolution required for some psychophysical experiments may be different from SVGA limits. This will require better goggles. It is worth noting that the image property in LCDs is optimized only for the native resolution and decreasing the resolution may lead to poorer image quality [58].

While performing experiments with high frequency stimuli, one should be very cautious about the response time of the liquid crystals used in the particular display. This leads to limited refresh frequency often causing image sticking and ghosting effects on the screens [59]. The limited contrast feature of the LCD display may also be a concern for some experiments. Yet another consideration while using these devices should be the uniformity of the stimulus. A non- 
uniform stimulus introduces several artefacts in functional imaging experiments as shown by Strasburger and coworkers using projectors [45, 46]. Additionally, LCD screens are prone to high non-uniformity if not designed properly $[60,61]$. Hence, artefacts similar to those reported by Strasburger may affect the imaging experiments with LCD goggles. This means that the display may require correction for spatial, contrast as well as spectral non-uniformity depending on the specific stimulus used in the experiments. However, any it is possible to calibrate the goggles outside the imaging experiment and hence there is no need for any complex fibre-optic calibration setup as that of Strasburger and co-workers [45].

We have listed some of the important technical considerations in choosing particular LCD goggles. However, the exact performance requirements will vary from experiment to experiment. The Video Electronics Standards Association (VESA) has suggested standards for commercial testing of LCD screens [62]. Test-beds for LCDs have also been designed by professional agencies [63]. Researchers may utilize these test-beds and methodology to characterize their goggles. It must be emphasized here that most of these test strategies have been designed for wide-angle viewing in varying illumination conditions. Hence, they might require improvements for testing in limited viewing angles and the fMRI experimental setups.

\section{Software Consideration}

The graphic card controlling the two goggles requires dedicated software for driving them. Additional software is also required to produce and display the stimuli. One strategy to design such experiments is to produce the stimulus frames with a graphics program, store them on a medium and use a different display program to access these frames and present them on the two screens. Commercial software like Presentation from Neurobehavioral Systems could be used for this purpose [64].

For our experiments, however, we developed an in-house software named Ananas. It uses Microsoft's DirectX 8 libraries and displays the two different stimuli on different screens, each with their own timing. The program was run on a Pentium IV processor machine with Windows 98 operating system. The performance in terms of time resolution, however, was found to be better on a machine running WindowsXP operating system.

The stimulus consisted of gratings of various kinds. These gratings were created internally by the program and were stored in an array of frames. Since the number of gratings required was limited in this experiment, they could be stored in the graphic card memory. If, however, the number of stimuli is high, one may have to use the system memory and use a prefetching technique to obtain optimal time requirement. In conditions where the required time resolution is even more demanding, real time operating systems may be preferred. From the requirements of these experiments, a grating was shown to either eye, while the other viewed the background screen. A central attention control task was presented identically on the two screens. The stimulus and the background screen were both stored as different frames in the graphic memory. Further, since simple gratings were used, they were generated at the beginning of the program from an input text file describing the conditions. The detailed documentation and the source code of this generate and display software are available freely from www.robots.ox.ac.uk/ bhaskar/fmri/ [65].

\section{CONCLUSION}

Dichoptic stimuli are an important tool in understanding the visual as well as some other brain functions. Traditionally, these have been generated using spectral or polarization filters and fused images displayed on one screen. This approach has been extended to functional MRI experiments with considerable ease. However, use of fused images and the optical assembly limits the usability of these approaches. Hence special purpose assemblies which do not fuse images have been devised. Despite some improvement, these assemblies still suffer from need of optical alignment and special purpose hardware. We have used general purpose biscreen video goggles, removing most of the drawbacks of other systems. We believe that the goggle based setup has the least inconveniences of all devices for visual dichoptic stimulation. It can be used for both binocular as well as dichoptic stimulation without any other optical assembly. Further, the stimuli types that can be presented using this setup are virtually infinite. Owing to ease of use and wide range of applications such goggle based devices have the potential of becoming the principal medium of visual stimulus presentation in future fMRI research. There are few concerning issues, however, which should be considered before employing these in experiments and hence further development and improvement is strongly encouraged. This includes development of standard calibration methods for such devices and improved display resolution.

\section{DECLARATION}

The authors have no commercial interest in the work presented in this paper and are not associated with any of the firms producing bi-screen goggles. The software from the authors for goggles is free and open-source; however, it comes with no warranty or support.

\section{ACKNOWLEDGEMENTS}

This work was supported by grants from the Deutsche Forschungsgemeinschaft to Ruxandra Sireteanu (Si 344/171, 2, 3). Author Bhaskar Choubey was supported by a Scatcherd fellowship from the University of Oxford.

\section{REFERENCES}

[1] Lumer ED, Friston KJ, Rees G. Neural correlates of perceptual rivalry in the human brain. Science 1998; 280(5371): 1930-4.

[2] Tong F, Nakayama K, Vaughan JT, Kanwisher N. Binocular rivalry and visual awareness in human extrastriate cortex. Neuron 1998; 21(4): 753-9.

[3] Polonsky A, Blake R, Braun J, Heeger DJ. Neuronal activity in human primary visual cortex correlates with perception during binocular rivalry. Nat Neurosci 2000; 3(11): 1153-9.

[4] Büchert M, Greenlee MW, Rutschmann RM, Kraemer FM, Luo F, Hennig J. Functional magnetic resonance imaging evidence for binocular interactions inhuman visual cortex. Exp Brain Res 2002; 145(3): 334-9.

[5] Muckli L, Jurcoane A, Choubey B, Kohler A, Singer W, Sireteanu R. Interocular transfer of orientation specific fMRI adaptation, 12th Annual Meeting of the Organization for Human Brain Mapping 2006. NeuroImage 2006; 31(Suppl 1): 134.

[6] Backus BT, Fleet DJ, Parker AJ, Heeger DJ. Human cortical activity correlates with stereoscopic depth perception. J Neurophysiol 2001; 86(4): 2054-68. 
[7] Nishida Y, Hayashi O, Iwami T, et al. Stereopsis-processing regions in the human parieto-occipital cortex. Neuroreport 2001; 12(10): 2259-63.

[8] Merboldt KD, Baudewig J, Treue S, Frahm J. Functional MRI of self-controlled stereoscopic depth perception. Neuroreport 2002; 13(14): 1721-5.

[9] Sireteanu R, Tonhausen N, Muckli L, Lanfermann H, Zanella F, Singer W. Cortical site of the amblyopic deficit in strabismic and anisometropic subjects, investigated with fMRI. Investig Ophthalmol Visual Sci 1998; 39: 909.

[10] Liu GT, Miki A, Goldsmith Z, et al. Eye dominance in the visual cortex using functional MRI at $1.5 \mathrm{~T}$ : an alternative method. J Am Assoc Pediatr Ophthalmol Strabismus 2002; 6(1): 40-8.

[11] Muckli L, Kiess S, Tonhausen N, Singer W, Goebel R, Sireteanu $\mathrm{R}$. Cerebral correlates of impaired grating perception in individual, psychophysically assessed human amblyopes. Vision Res 2006; 46(4): 506-26.

[12] Jurcoane A, Choubey B, Muckli L, Sireteanu R. A pilot study for investigating cortical binocularity in humans using fMRI adaptation. Strabismus 2007; 15(1): 33-7.

[13] Wade N. A Natural History of Vision. MIT Press, Cambridge, Massachusetts 1998.

[14] Tsao DY, Vanduffel W, Sasaki Y, et al. Stereopsis activates V3A and caudal intraparietal areas in macaques and humans. Neuron 2003; 39(3): 555-68.

[15] Tyler CW, Likova LT, Kontsevich LL, Wade AR. The specificity of cortical region KO to depth structure. Neuroimage 2006; 30(1): 228-38.

[16] Moutoussis K, Keliris G, Kourtzi Z, Logothetis N. A binocular rivalry study of motion perception in the human brain. Vision Res 2005; 45(17): 2231-43.

[17] Haynes JD, Rees G. Predicting the stream of consciousness from activity in human visual cortex. Curr Biol 2005; 15(14): 1301-7.

[18] Miki A, Liu GT, Goldsmith ZG, Liu CSJ, Haselgrove JC. Decreased activation of the lateral geniculate nucleus in a patient with anisometropic amblyopia demonstrated by functional magnetic resonance imaging. Ophthalmologica 2003; 217(5): 365-9.

[19] Gilaie-Dotan S, Ullman S, Kushnir T, Malach R. Shape-selective stereo processing in human object-related visual areas. Hum Brain Mapp 2002; 15(2): 67-79.

[20] Williams MA, Morris AP, McGlone F, Abbott DF, Mattingley JB. Amygdala responses to fearful and happy facial expressions under conditions of binocular suppression. J Neurosci 2004; 24(12): 2898-904.

[21] Neri P, Bridge H, Heeger DJ. Stereoscopic processing of absolute and relative disparity in human visual cortex. J Neurophysiol 2004; 92: 1880-91.

[22] Cornelissen FW, Pelli DG, Farell B, Huckins SC, Szeverenyi NM. A binocular fiberscope for presenting visual stimuli during fMRI. Spat Vis 1997; 11(1): 75-81.

[23] Jurcoane A, Muckli L, Choubey B, Sireteanu R. Cortical binocularity in human stereoblindness: A study with functional magnetic resonance imaging. Perception 2006; 35: ECVP Abstract (Suppl).

[24] NordicNeuroLab. Presenting Visual stimulus in fMRI. Bergen, Norway. http://www.nordicneurolab.com/

[25] Resonance Technologies. VisuaStimDigital for fMRI Resonance Technologies, Northrige, USA. http://www.mrivideo.com

[26] Schmitz B, Käsmann-Kellner B, Schäfer T, et al. Monocular visual activation patterns in albinism as revealed by functional magnetic resonance imaging. Hum Brain Mapp 2004; 23(1): 40-52.

[27] Brouwer G, van Ee R, Schwarzbach J. Activation in visual cortex correlates with the awareness of stereoscopic depth. J Neurosci 2005; 25(45): 10403-13.

[28] Fang F, He S. Cortical responses to invisible objects in the human dorsal and ventral pathways. Nat Neurosci 2005; 8(8): 1380-5.

[29] Wunderlich K, Schneider KA, Kastner S. Neuralcorrelates of binocular rivalry in the human lateral geniculate nucleus. Nat Neurosci 2005; 8(11): 1595-602.

[30] Negawa T, Mizuno S, Hahashi T, et al. M pathway and areas 44 and 45 are involved in stereoscopic recognition based on binocular disparity. Jpn J Physiol 2002; 52(2): 191-8.

[31] Pasley BN, Mayes LC, Schultz RT. Subcortical discrimination of unperceived objects during binocular rivalry. Neuron 2004; 42(1): 163-72.
[32] Haynes JD, Deichmann R, Rees G. Eye-specific effects of binocular rivalry in the human lateral geniculate nucleus. Nature 2005; 438: 496-9.

[33] Naganuma T, Nose I, Inoue K, Takemoto A, Katsuyama N, Taira M. Information processing of geometrical features of a surface based on binocular disparity cues: an fMRI study. Neurosci Res 2005; 51(2): 147-55.

[34] Tong F, Engel SA. Interocular rivalry revealed in the human cortical blind-spot representation. Nature 2001; 411: 195-9.

[35] Lee $\mathrm{SH}$, Blake R. V1 activity is reduced during binocular rivalry. J Vis 2002; 2(9): 618-26.

[36] Moutoussis K, Zeki S. The relationship between cortical activation and perception investigated with invisible stimuli. Proc Natl Acad Sci USA 2002; 99(14): 9527-32

[37] Meng M, Remus DA, Tong F. Filling-in of visual phantoms in the human brain. Nat Neurosci 2005; 8(9): 1248-54

[38] Gulyás B, Roland PE. Binocular disparity discrimination in human cerebral cortex: functional anatomy by positron emission tomography. Proc Natl Acad Sci USA 1994; 91(4): 1239-43.

[39] Rutschmann RM, Schrauf M, Greenlee MW. Brain activation during dichoptic presentation of optic flow stimuli. Exp Brain Res 2000; 134(4): 533-7.

[40] Rutschmann RM, Greenlee MW. BOLD response in dorsal areas varies with relative disparity level. Neuroreport 2004; 15(4): 615-9.

[41] Rutschmann RM, Büchert M, Hennig J, Greenlee MW. Binocular interactions in human visual cortex: evidence from fMRI. Perception 2002; 31: ECVP (Abstract Suppl).

[42] Taira M, Tsutsui KI, Jiang M, Yara K, Sakata H. Parietal neurons represent surface orientation from the gradient of binocular disparity. J Neurophysiol 2000; 83(5): 3140-6.

[43] Kwak Y, MacDonald L. Characterisation of a desktop LCD projector. Displays 2000; 21(5): 179-94.

[44] Majumder A, Stevens R. Color nonuniformity in projection-based displays: Analysis and solutions. IEEE Trans Vis Comput Graph 2004; 10(2): 177-88

[45] Strasburger H, Wüstenberg T, Jäncke L. Calibrated LCD/TFT stimulus presentation for visual psychophysics in fMRI. J Neurosci Methods 2002; 121(1): 103-10.

[46] Strasburger H, Wüstenberg T. Calibrated LCD stimulus presentation for visual psychophysics in fMRI. In $6^{\text {th }}$ Annual Meeting of the Vision Sciences Society 2006.

[47] Wiens S, Fransson P, Dietrich T, Lohmann P, Ingvar M, Ohman A. Keeping it short: a comparison of methods for brief picture presentation. Psychol Sci 2004; 15(4): 282-5.

[48] Wiens S, Ohman A. Visual masking in magnetic resonance imaging. Neuroimage 2005; 27(2): 465-7.

[49] Iwami T, Nishida Y, Hayashi O, et al. Common neural processing regions for dynamic and static stereopsis in human parieto-occipital cortices. Neurosci Lett 2002; 327(1): 29-32.

[50] Nishida Y, Hayashi O, Iwami T, Kimura M, Kani K, Ito R. Development of a new binocular visual stimulation device using image guides for functional mri. Neuro Ophthalmol 2000; 24(2): 343-8.

[51] Fibre Optic Visual System, Avotec, Florida, USA. http://www.avotec.org

[52] Logothetis NK, Sheinberg DL. Visual object recognition. Annu Rev Neurosci 1996; 19: 577-21.

[53] Lee S, Blake R, Heeger DJ. Traveling waves of activity in primary visual cortex during binocular rivalry. Nat Neurosci 2004; 8: 22-3.

[54] Hamann S, Herman RA, Nolan CL, Wallen K. Men and women differ in amygdala response to visual sexual stimuli. Nat Neurosci 2004; 7(4): 411-6.

[55] Gardner JL, Sun P, Waggoner RA, Ueno K, Tanaka K, Cheng K. Contrast adaptation and representation in human early visual cortex. Neuron 2005; 47(4): 607-20.

[56] James TW, Gauthier I. Repetition-induced changes in bold response reflect accumulation of neural activity. Hum Brain Mapp 2005; 27(1): 37-46.

[57] Engström M, Ragnehed M, Lundberg P. Projection screen or video goggles as stimulus modality in functional magnetic resonance imaging. Magn Reson Imaging 2005; 23(5): 695-9.

[58] Cushman W. Comparison of CRT vs. LCD monitors for desktop Computers, ErgoSense Issue 13, The Ergonomics Consortium, Milwaukee, WI, USA. http://www.details-worktools.com/media/ scms/ISSUE-13.pdf 
[59] Don-Gyou L, Il-Ho K, Soh SHS, Byung-Chul A. A measurement and analysis method of image sticking in LCD. SID Symp Digest Tech Papers 2002; 33(1): 324-7.

[60] Sotokawa A, Ishibashi O. LCD technology. Fujitsu Sci Tech J (FSTJ): Mobile Comput 1998; 34(1): 107-11.

[61] Tamura T, Satoh T, Uchida T, Furihata T. Quantitative evaluation of luminance nonuniformity mura in LCDs based on just noticeable difference (JND) contrast at various background luminances. IEICE Trans Electron 2006; E89-C(10): 1435-40.
[62] Video Electronics Standards Association, VESA Flat Panel display measurement standard. 2nd ed. 2005; http://www.vesa.org/Public/ Fpdm2/

[63] CNET Labs. How we test : LCD monitors http://reviews.cnet.com/ 4520-6603_7-5098394-1.html

[64] Neurobehavioral Systems, Presentation ${ }^{\odot}$ software, http://www. neurobs.com/presentation

[65] Choubey B. Ananas User guide and Source Code documentation 2006; http://www.robots.ox.ac.uk/ bhaskar/fmri/

(C) Choubey et al.; Licensee Bentham Open.

This is an open access article licensed under the terms of the Creative Commons Attribution Non-Commercial License (http://creativecommons.org/licenses/by-nc/3.0/) which permits unrestricted, non-commercial use, distribution and reproduction in any medium, provided the work is properly cited. 